\title{
Extracellular Vesicles Support the Survival of Serum- dependent cells in vitro
}

\author{
Christy Mikhael, Shannon Holliday \& Guanghong Han \\ University of Florida
}

Faculty mentor: Shannon Holliday, College of Dentistry

\begin{abstract}
Extracellular vesicles (EVs) are $30-150 \mathrm{~nm}$ in diameter and are released by cells into the extracellular environment. They facilitate intercellular communication and may be involved in cell survival. Although serum-dependent cells are not obviously affected by depletion of EVs from the serum, we previously reported that cells in serum that contain EVs were more resistant to the drugs enoxacin and bis-enoxacin, compared to cells in serum without EVs. This change in resistance led us to examine whether EVs are sufficient to maintain serum dependent cells in vitro. Here, we tested the effect of EVs on the survival RAW 264.7 cells and on 4T1 murine breast cancer cells. EVs were isolated by differential centrifugation and were counted over various time periods. Cells were grown in alpha or Dulbecco's minimal essential media supplemented with FBS, EVs from various sources, or with no supplements. Cell growth was determined by cell counts. In media without supplementation, the RAW 264.7 cells and 4T1 cells died by three days. Media supplemented with EVs from FBS allowed the cells to survive in the absence of FBS for one week or more. Being able to perform experiments on cells supported by EVs will simplify the interpretation of experiments. Efforts are underway to determine their mechanism by which EVs support cell survival.
\end{abstract}

Keywords: Extracellular vesicle, FBS, serum

\section{Introduction}

Extracellular vesicles (EVs) are 30-150 nm in diameter and are released by cells into the extracellular environment (Yáñez-Mó et al., 2015). The term EVs covers exosomes and microvesicles. Although both exosomes and microvesicles play multiple biological roles when it comes to intercellular communication and other physiological processes, there are differences.

Exosomes are released from cells towards the end of the endocytic pathway when multivesicular bodies fuse with the plasma membrane. On the other hand, microvesicles are heterogenous in nature, and are created by budding off directly from the plasma membrane (Lee, Andaloussi, \& Wood, 2012). Initially thought to be the "garbage disposals" of endocytic origin.

During the past decade, a large amount of data indicating the exosomes function in intercellular signaling were generated and are available. They contain receptors that can stimulate ligands on target cells to stimulate cell signaling. For example, recently, it was shown 
the receptor activator of nuclear factor kappa B-ligand (RANKL) and its receptor RANK are both present in exosomes and serve vital functions in regulating bone remodeling (Holliday et al, 2017). Additionally, exosomes can fuse with target cells to deliver luminal contents, such as functional microRNAs (Lee, Andaloussi, \& Wood, 2012). This means that exosomes have the capcity to regulate target cells both through traditional cell signaling pathways, and by altering protein translation (Valadi et al, 2007). Taken together, this suggests the idea that exosomes may be particularly potent intercellular regulators. Emerging data has supported that hypothesis (Yáñez-Mó et al., 2015). Additionally, EVs contain various proteins and nucleic acids such as mRNA and microRNAs (Holliday et al, 2017).

RAW 264.7 cells are a murine macrophage cell line that functions as a precursor to osteoclasts. Osteoclasts (OC) are multinucleated cells that are derived from cell differentiation via osteoclastogenesis. The murine breast cancer cell line, 4T1, has often been used to study how breast cancer cells invade bone. Both cell lines are serum-dependent cells. In previous studies, it was determined that cells in serum that contained EVs were more resistant to the drugs, enoxacin and bis-enoxacin compared to cells grown in serum without EVs (Vracar et al., 2018). We sought to test whether EVs are sufficient to maintain serum dependent cells in vitro.

\section{Methods}

\section{Reagents}

Dulbecco's minimum essential media (dMEM) and minimum essential media, $\alpha$ modification $(\alpha \mathrm{MEM})$ were obtained from Sigma/Aldrich Chemical CO (St. Louis MO, USA). ExoQuick TC was obtained from Systems Biosciences (Mountain View CA, USA). Phosphate-buffered saline (PBS) and penicillin/streptomycin were purchased from Corning (Manassas, VA, USA).

\section{Cell Culture}

4T1 murine breast cancer cells were cultured using dMEM supplemented with $10 \%$ fetal bovine serum (FBS), 1\% L-glutamine (Thermo Fisher Scientific, Waltham, MA, USA), and 1\% penicillin /streptomycin /amphotericin B (Thermo Fisher). RAW 264.7 cells were cultured in a similar fashion, cultured using aMEM supplemented with $10 \%$ fetal bovine serum (FBS), 1\% Lglutamine (Thermo Fisher Scientific, Waltham, MA, USA), and 1\% penicillin /streptomycin /amphotericin B (Thermo Fisher). Cells were grown in an incubator at 37 degrees Celsius ( $5 \%$ $\mathrm{CO}_{2}$ ). Aftwerwards, cells were cultured in different conditions. The RAW 264.7 cells and 4T1 
cells were supplemented with $10 \% \mathrm{FBS}, 10 \% \mathrm{FBS}$ with no EVs, EVs from FBS $(5 \mathrm{X} \mathrm{10} / \mathrm{ml})$ or no supplement at all. Media was replaced every three days.

\section{Isolation of EVs}

Isolation of EVs were done in sterile conditions. To isolate EVs from FBS, serum was spun at low speed $(10,000 \mathrm{X} g$ ) for 20 minutes. The supernatant was collected and spun at 100,000 X $g$ for 4 hours in a Beckman LM8 unltracentrifuge, the pellet was resuspended in phosphate buffered saline (PBS) at a concentration of 1 X $10^{9} \mathrm{EVs} / \mathrm{ml}$ by Exocet (Systems Biosciences).

\section{Cell counting}

Cells were counted by volunteers who had been calibrated and were blinded to treatment conditions. Cells were counted in three random locations $(1000 \mu \mathrm{m} \times 1000 \mu \mathrm{m}$ square $)$ per well.

\section{Results}

Long term survival on both 4T1 cells and RAW 264.7 cells were tested. In Figure 1, we see the effect of exosomes harvested from FBS on the RAW 264.7 cells. It is shown that cells in the FBS and FBS with no EVs conditions died after three days. Similar results were present for the no supplement condition as well. However, the results for the response to EVs from FBS show that the cells were able to survive for eleven days. Under these conditions, cell proliferation was very slow, but the cells appeared viable for the long culture period.

Similar results were obtained with the $4 \mathrm{~T} 1$ cell line. In Figure 2, we see the effect of exosomes harvested from FBS on the 4T1 cells. it is shown that cells in the conditions of FBS, FBS with no EVs, and no supplement, died within four days. The condition with no supplement also died within a comparable time frame of four days. The EVs from FBS condition showed a slow cell proliferation rate over a culture period of eight days. 


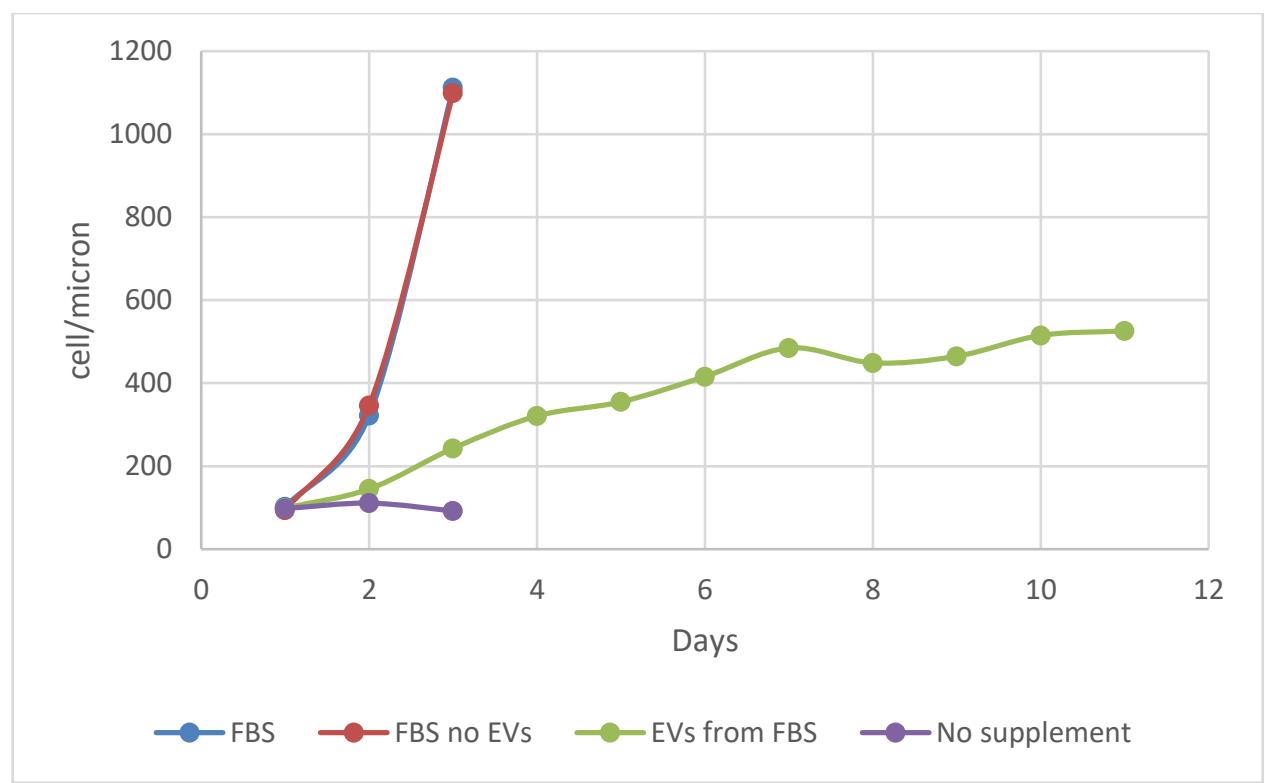

Figure 1. Long term survival study of RAW 264.7 cells in $\alpha$ MEM supplement with EVs from FBS

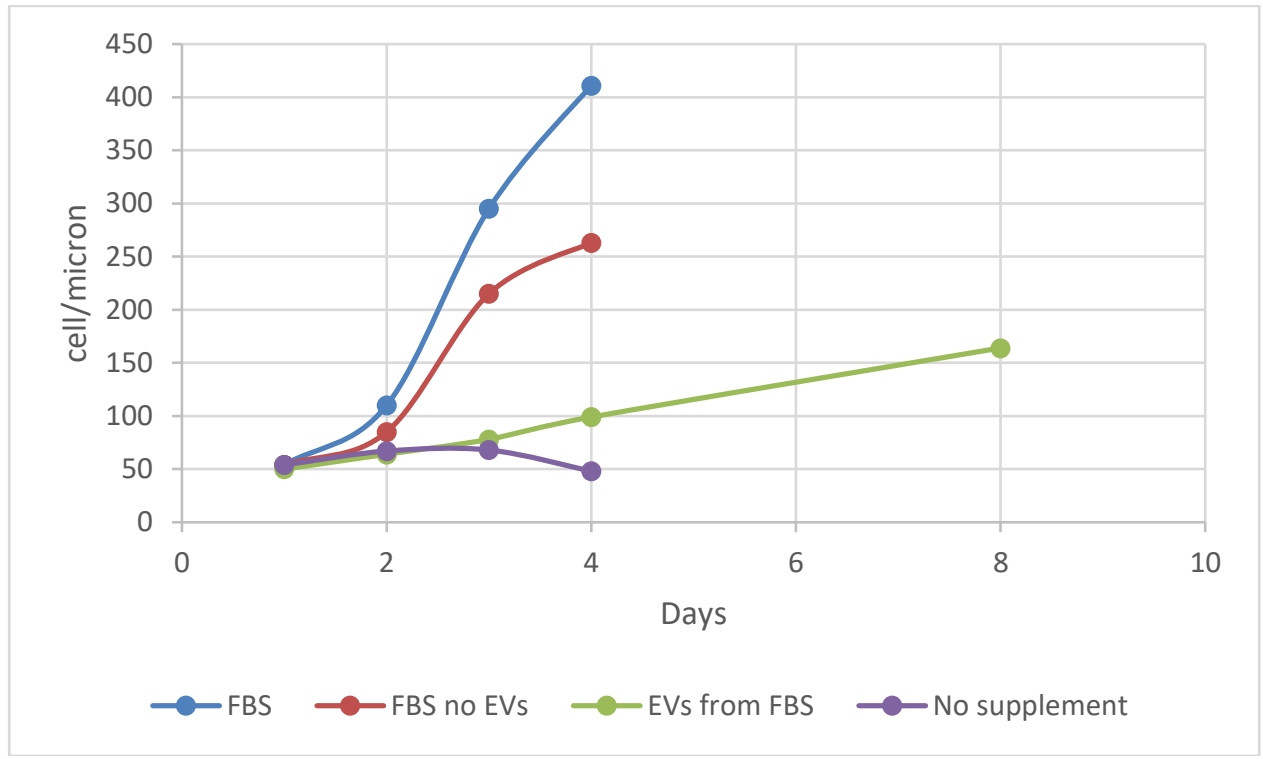

Figure 2. Long term survival study of $4 \mathrm{~T} 1$ cells in dMEM supplement with EVs from FBS

\section{Discussion}

The RAW 264.7 cells in the FBS and FBS with no EVs conditions died after the three day period due to the fact that FBS has factors that stimulate cell growth, instigating the cells to overgrow the plate, causing them to die. This was also the case for no supplement, but rather than dying because of overgrowth, the cells died due to the fact that they had no nutrients to support them. 
Similar reasons are behind the death of the 4T1 cells under the FBS and FBS with no EVs conditions. The growth factors present in FBS stimulated cell growth, causing the cells to overgrow their plate. The no supplement condition may have survived for the same amount of time, but this was due to lack of nutrients in the system, unable to provide a setting that stimulates growth/proliferation.

For the first time, here we are able to show that EVs alone can support the survival of serumdependent cells. This is a potentially important development since many cells cannot be grown in a defined media. Fetal Bovine Serum (FBS) is widely used in cell biology, and is essential for the growth and survival of many cell types. Derived from fetal calves, FBS is a complex "black box" that is required by cells, but the mechanism(s) by which it functions are not known. Thus, the results of most cell culture experiments must be interpreted in the context of the unknown activities provided by FBS. Worse still, different lots of FBS often produce different effects.

\section{Conclusion}

The use of EVs rather than FBS may allow clearer interpretation of many types of experiments. For example, phosphorylation assays are often performed with serum-dependent cells in serum-free media. Serum stimulates many signaling pathways making it difficult to detect the effects of specific factors. By necessity, such experiments are done over short time frames, and there is concern regarding whether the lack of nutrients provided by serum affects the assay. Phosphorylation assays can, in principle, be performed in EVs over longer periods of time under better defined condtions.

It may prove to be very useful to examine the mechanism by which EVs promote cell survival. Is it by providing specific nutrients, and are signaling pathways triggered? It is also of interest to determine whether the EVs fuse with the cells to deliver elements to the cells' membranes and/or cytosol.

The current study is limited by the use of EVs from only one source and only two types of cells. This study also relied of cell counting which may be susceptible to mistaking dead cells for viable cells and vice versa. Finally, the current study only used one method for isolating EVs. It is possible that contaminants in the EVs preprations are affecting the cell survival. Despite these limitations, the current report strongly supports the hypothesis that EVs, by themselves, are sufficient to promote cell survival. 


\section{References}

Holliday, L. S., McHugh, K. P., Zuo, J., Aguirre, J. I., Neubert, J. K., \& Rody, W. J. (2017). Exosomes: novel regulators of bone remodelling and potential therapeutic agents for orthodontics. Orthodontics \& craniofacial research, 20 Suppl 1(Suppl 1), 95-99. doi:10.1111/ocr.12165

Huynh, N., VonMoss, L., Smith, D., Rahman, I., Felemban, M. F., Zuo, J., Holliday, L. S. (2016). Characterization of Regulatory Extracellular Vesicles from Osteoclasts. Journal of dental research, 95(6), 673-679. doi:10.1177/0022034516633189

Lee Y., El Andaloussi S., \& Wood M., (2012). Exosomes and microvesicles: extracellular vesicles for genetic information transfer and gene therapy, Human Molecular Genetics, 21(R1), R125R134, doi:10.1093/hmg/dds317

Valadi H, Ekstrom K, Bossios A, Sjostrand M, Lee J, \& Lötvall J. (2007). Exosome-mediated transfer of mRNAs and microRNAs is a novel mechanism of genetic exchange between cells. Nat Cell Biol. 9:654-659. doi: 10.1038/ncb1596

Vracar, T. C., Zuo, J., Park, J., Azer, D., Mikhael, C., Holliday, S. A., Holliday, L. S. (2018). Enoxacin and bis-enoxacin stimulate 4T1 murine breast cancer cells to release extracellular vesicles that inhibit osteoclastogenesis. Scientific reports, 8(1), 16182. doi:10.1038/s41598-018-34698-9

Yáñez-Mó, M., Siljander, P. R., Andreu, Z., Zavec, A. B., Borràs, F. E., Buzas, E. I., De Wever, O. (2015). Biological properties of extracellular vesicles and their physiological functions. Journal of extracellular vesicles, 4, 27066. doi:10.3402/jev.v4.27066 